\title{
Amplification of Papillomavirus Oncogene Transcripts Assay for Bowenoid Papulosis
}

\author{
Takahide Kaneko $^{1 *}$, Gen Nakahishi ${ }^{2}$, Koji Nakajima ${ }^{1}$, Takayuki Aizu ${ }^{1}$, Kayo Jin ${ }^{1}$, Daiki Rokunohe ${ }^{1}$, \\ Yasushi Matsuzaki ${ }^{1}$, Nakano Hajime ${ }^{1}$, Toshihiro Tanaka ${ }^{2}$, Daisuke Sawamura ${ }^{1}$ \\ ${ }^{1}$ Department of Dermatology, Hirosaki University Graduate School of Medicine, Hirosaki, Japan; ${ }^{2}$ Department of Dermatology, \\ Shiga University of Medical Science, Otsu, Japan. \\ Email: *derma@cc.hirosaki-u.ac.jp
}

Received July 31 ${ }^{\text {st }}, 2013$; revised August 20 ${ }^{\text {th }}, 2013$; accepted September $23^{\text {rd }}, 2013$

Copyright (C) 2013 Takahide Kaneko et al. This is an open access article distributed under the Creative Commons Attribution License, which permits unrestricted use, distribution, and reproduction in any medium, provided the original work is properly cited.

\begin{abstract}
Human papillomavirus (HPV) is an important causative agent of cervical carcinoma and some malignant cutaneous tumors. The integration of HPV DNA into the host genome is one of the most important risk factors for malignant transformation. Here, we report the case of a 53-year-old man with multiple, black-brown genital macules and nodules. Histological findings resembled those for Bowen's disease, and we made a diagnosis of bowenoid papulosis. This condition is generally benign, but invasive squamous cell carcinoma (SCC) arising from bowenoid papulosis has been previously reported. Therefore, we took biopsy specimens from 10 lesions and tested them for the presence of HPV DNA. DNA sequencing identified HPV type 16 (HPV-16) DNA in all samples. Next, we excised 4 different relatively large lesions and performed an amplification of papillomavirus oncogene transcripts (APOT) assay. This assay showed that all samples had only episome-derived viral transcripts, indicating no integration of HPV DNA into the host genome. We have followed this case for 3 years, and no progression to Bowen's disease or SCC has so far been observed. We conclude that the APOT assay is a feasible method for evaluating the malignant potential of bowenoid papulosis.
\end{abstract}

Keywords: HPV 16; Integration; Malignant Transformation

\section{Introduction}

Human papillomavirus (HPV) infection of the genital skin shows a variety of presentations ranging from benign condyloma acuminata to squamous cell carcinoma (SCC) $[1,2]$. Bowenoid papulosis is a condition that histologically resembles Bowen's disease and manifests small verrucous papules and nodules on the genitalia [3]. The condition is benign and has a tendency towards spontaneous resolution. The high-risk, oncogenic HPV type 16 (HPV-16) has been detected in most cases, and invasive SCC arising from bowenoid papulosis has been described in some cases [4]. Here we report the case of a 53-year-old man with bowenoid papulosis in which the amplification of papillomavirus oncogene transcripts (APOT) assay served as a feasible method for evaluating prognosis.

*The authors have no conflict of interest to declare.

${ }^{\#}$ Corresponding author.

\section{Materials and Methods}

\subsection{Patient}

A 53-year-old man presented with multiple skin lesions on the genital area. The lesions were first noticed 3 years earlier. His medical history included diabetes mellitus for 3 years and hypertension for 5 years. His family history was unremarkable. Physical examination revealed multiple, keratotic, black-brown macules and nodules 0.5 - 3.0 $\mathrm{cm}$ in diameter on his genitalia (Figure 1). Inguinal lymph nodes were not palpable. Skin biopsies were taken from 2 lesions, including both nodule and plaque. Histological findings from the nodule and plaque showed a variable extent of hyperkeratosis, irregular acanthosis, papillomatosis, and cytological atypia with few dyskeratotic cells in the entire upper epidermis (Figure 2).

\subsection{Immunohistochemical Study}

Skin sections were fixed in $10 \%$ formaldehyde and 


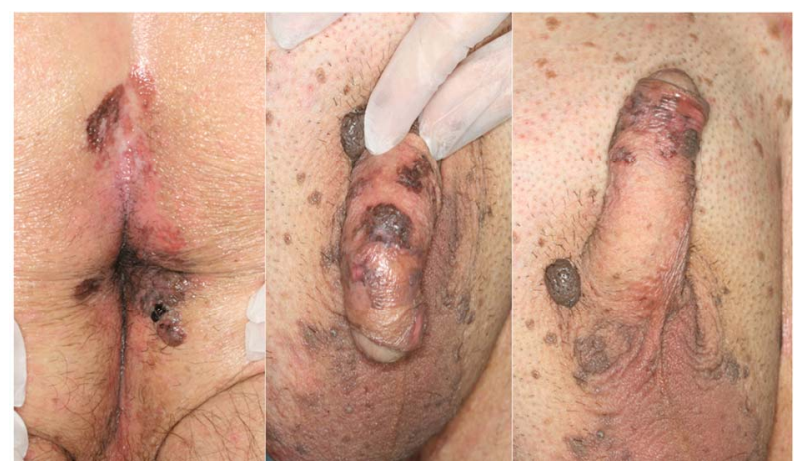

Figure 1. Clinical findings. Multiple, keratotic, black-brown macules and nodules $0.5-3.0 \mathrm{~cm}$ in diameter on the patient's genitalia.

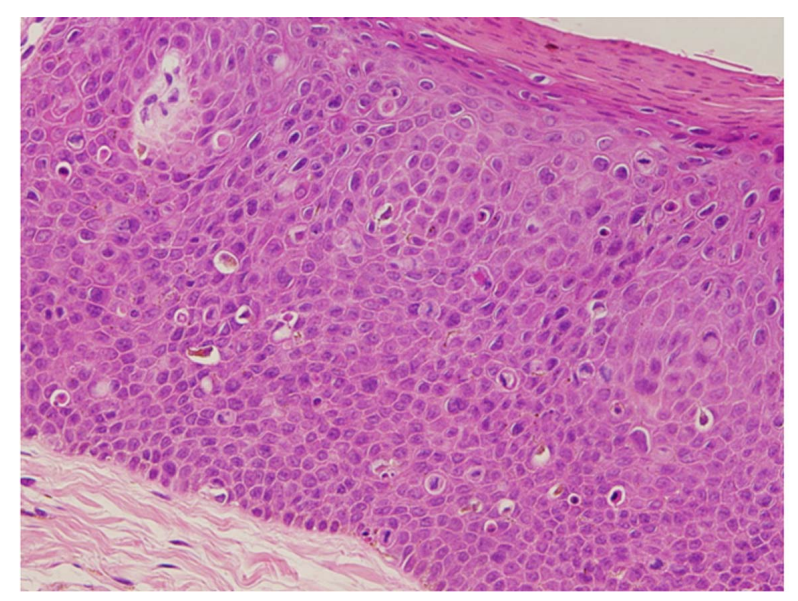

Figure 2. Histological findings. Variable extent of hyperkeratosis, irregular acanthosis, papillomatosis, and cytological atypia with few dyskeratotic cells in the entire upper epidermis.

embedded in paraffin. The sections were routinely passed through xylene and a graded alcohol series, and stained with several antibodies using the avidin-biotin-peroxidase complex method. Peroxidase binding was detected using the diaminobenzidine method, and the sections were then lightly counterstained with hematoxylin for microscopic examination.

\subsection{HPV Typing and APOT Assay}

Total DNA was extracted from the lesions and HPV DNA was amplified using GP5/GP6 primers. The amplification products were sequenced using an ABI 3100 sequencer.

Total RNA was isolated and reverse transcribed using a commercial kit (Superscript III First-Strand Synthesis System; Invitrogen Corp., Carlsbad, CA, USA) and an oligo-(dT) $)_{17}$ primer coupled to a linker sequence. PCR amplification for the APOT assay was carried out as previously described $[5,6]$. APOT products were visualized by performing agarose gel electrophoresis, excised from the gel, and sequenced using an ABI 3100 sequencer. G3PDH transcript was used as control.

\section{Results}

\subsection{Histologic Detection of HPV}

First, we performed an immunohistochemical analysis using the anti-HPV capsid antigen antibody, K1H8 (DAKO, Tokyo, Japan). The analysis indicated that some epidermal keratinocytes were HPV-positive (data not shown). After confirmation of HPV infection, we proceeded to further HPV analysis.

\subsection{Identification of $\mathrm{HPV}-16$}

Next, we extracted total DNA from biopsy specimens taken from 10 pigmented lesions. We performed PCR amplification using GP5/GP6 primers targeting the HPV L1 gene and sequenced the PCR products. After alignment of our sequences with HPV L1 sequences obtained in GenBank, conserved sequence regions were selected and analyzed using a computer software program. We identified sequences corresponding with HPV-16 in all 10 samples.

\subsection{APOT Assay Detected Only Episome-Derived Viral Transcripts}

To know the extent of the integration of HPV into the patient's genome, we performed an APOT assay [5-7]. We excised 4, relatively large different lesions. Total RNA was isolated and reverse transcribed. APOT products were visualized by agarose gel electrophoresis. The result was shown in Figure 3. PCR amplification detected the bands corresponding to the episomal form of HPV-16 transcript in samples 1, 2 and 4 while sample 3 showed no clear band. Also we found an additional short band in sample 4 , the size of which was predicted to be integrated form. Sequence analysis of those bands revealed that all larger band (black arrow in Figure) had only episome-derived viral transcripts and the smaller band (white arrow in Figure 3) was also originated from the viral transcript, indicating no integration of the HPV DNA into the host genome (Figure 3 ). Host DNA could not be found by sequence analysis.

We have followed this case for 3 years and so far have observed no progression to Bowen's disease or SCC.

\section{Discussion}

Our patient showed multiple pigmented genital macules and nodules, and our clinical diagnosis was bowenoid papulosis. Histological findings were compatible to those for Bowen's disease and indicated bowenoid papulosis.

$\mathrm{HPV}$ is an etiological agent in cervical carcinomas and 


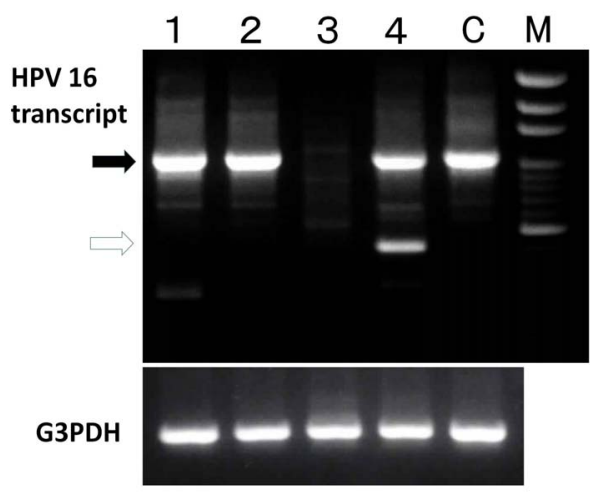

Figure 3. Amplification of papillomavirus oncogene transcripts (APOT) assay. Total RNA was isolated from 4 relatively large different lesions and reverse transcribed. APOT products were visualized by agarose gel. All samples were numbered from 1 to 4 . C: control sample from episomal transcript. M: DNA maker. G3PDH was used as control. PCR amplification detected the bands corresponding to the episomal form of HPV-16 transcript in samples 1, 2 and 4 (black arrow) while sample 3 showed no clear band. Also we found an additional short band (white arrow) in sample 4.

causes various types of malignant and benign skin tumors, including SCC, Bowen's disease, and bowenoid papulosis [1-3]. Bowenoid papulosis is benign, although HPV-16 has been detected in most cases. In our case, we took DNA from 10 lesions and detected HPV-16 in all samples. However, SCC arising from a bowenoid papulosis has been described in some cases [4].

Persistent infection with high-risk HPV types, such as HPV-16, 18, 31, 33, and 45, is necessary but insufficient for cervical carcinoma development. Integration of the HPV DNA into the host genome is one of the most important risk factors for malignant transformation [8]. The APOT assay can detect transcriptionally active virus-host fusion transcripts and distinguish between episomal and integrated HPV DNA [6]. In addition, the assay can determine the precise integration site of the viral genome in host chromosomes. The APOT assay requires only a small tissue sample and allows for the analysis of integration sites in multiple locations on the lesion.

Recently, we reported the case of a patient with multiple black plaques and an erythematous nodule on the vulva [7]. On histological examination, multifocal vulvar Bowen's disease and invasive SCC were found. APOT assay showed that 2 locations of Bowen's disease had only episome-derived HPV-16 transcripts, but another 2 sites of Bowen's disease and the nodule of invasive SCC had HPV-16 transcripts derived from integration.

We therefore performed an APOT assay about sample form 4 lesions in the present case. The result showed that samples 1 and 2 showed only the bands from episomederived HPV-16 transcripts. No band was observed in the lane of sample 3, but we did not know the reason. Also sample 4 showed the larger and smaller bands. The former band was corresponding to that from the episomal form. The smaller band was thought to be the band from the integrated form, but sequence analysis could detect only HPV-16 transcript without host sequence. We concluded that there was no integration of viral genome to host genome in this case. In fact, a 3-year follow-up did not show transformation to SCC. We suggest that the APOT assay is a feasible method for evaluating the clinical degree of malignancy in bowenoid papulosis.

\section{REFERENCES}

[1] S. Majewski and S. Jablonska, "Human PapillomavirusAssociated Tumors of the Skin and Mucosa," Journal of the American Academy of Dermatology, Vol. 36, No. 5, 1997, pp. 659-685.

http://dx.doi.org/10.1016/S0190-9622(97)80315-5

[2] T. M. Darragh, T. J. Colgan, J. Thomas Cox, D. S. Heller, M. R. Henry, R. D. Luff, T. R. Nayar, J. M. Palefsky, M. H. Stoler, E. J. Wilkinson, R. J. Zaino and D. C. Wilbur, "The Lower Anogenital Squamous Terminology Standardization Project for HPV-Associated Lesions: Background and Consensus Recommendations from the College of American Pathologists and the American Society for Colposcopy and Cervical Pathology," The International Journal of Gynecological Pathology, Vol. 32, No. 1, 2013, pp. 76-115. http://dx.doi.org/10.1097/PGP.0b013e31826916c7

[3] C. J. Herquet, "Anogenital Malignancies and Pre-Malignancies," Journal of the European Academy of Dermatology and Venereology, Vol. 25, No. 8, 2011, pp. 885895.

[4] A. Yoneta, T. Yamashita, H. Y. Jin, A. Iwasawa, S. Kondo and K. Jimbow, "Development of Squamous Cell Carcinoma by Two High-Risk Human Papillomaviruses (HPVs), a Novel HPV-67 and HPV-31 from Bowenoid Papulosis,” British Journal of Dermatology, Vol. 143, No, 3, 2000, pp. 604-608. http://dx.doi.org/10.1111/j.1365-2133.2000.03718.x

[5] G. Nakanishi, O. Yamasaki, Y. Nagao and T. Tanaka, "Detection of Human Papillomavirus Type 67 from Bowenoid Papulosis,” European Journal of Dermatology, Vol. 20, No. 6, 2010, pp. 819-820.

[6] R. Klaes, S. M. Woerner, R. Ridder, N. Wentzensen, M. Duerst, A. Schneider, B. Lotz, P. Melsheimer and M. von Knebel Doeberitz, "Detection of High-Risk Cervical Intraepithelial Neoplasia and Cervical Cancer by Amplification of Transcripts Derived from Integrated Papillomavirus Oncogenes," Cancer Research, Vol. 59, No. 24, 1999, pp. 6132-6136.

[7] G. Nakanishi, K. Fujii, K. Asagoe, T. Tanaka T and K. Iwatsuki, "Human Papillomavirus Genome Integration in Multifocal Vulvar Bowen's Disease and Squamous Cell Carcinoma," Clinical and Experimental Dermatology, Vol. 34, No. 8, 2009, pp. e965-967.

http://dx.doi.org/10.1111/j.1365-2230.2009.03708.x 
[8] I. Kraus, C. Driesch, S. Vinokurova, E. Hovig, A. Schneider, M. von Knebel Doeberitz and M. Duerst, "The Majority of Viral-Cellular Fusion Transcripts in Cervical Carcinomas Cotranscribe Cellular Sequences of Known or Predicted Genes,” Cancer Research, Vol. 68, No. 7, 2008, pp. 2514-2522.

http://dx.doi.org/10.1158/0008-5472.CAN-07-2776 